\title{
Reduction of protein kinase MARK4 in the brains of experimental scrapie rodents and human prion disease correlates with deposits of $\operatorname{PrP}^{\mathrm{Sc}}$
}

\author{
HAN-SHI GONG ${ }^{1,2}$, YAN GUO ${ }^{2}$, CHAN TIAN $^{2}$, WU-LING XIE ${ }^{1,2}$, QI SHI ${ }^{2}$, JIN ZHANG $^{2}$, YIN XU $^{2}$, \\ SHAO-BIN WANG ${ }^{2}$, BAO-YUN ZHANG $^{2}, \mathrm{CAO} \mathrm{CHEN}^{2}$, YONG LIU $^{1}$ and XIAO-PING DONG ${ }^{2}$ \\ ${ }^{1}$ School of Medicine, Xi'an Jiao Tong University, Xi'an 710061; ${ }^{2}$ State Key Laboratory for Infectious Disease \\ Prevention and Control, National Institute for Viral Disease Control and Prevention, Chinese \\ Center for Disease Control and Prevention, Beijing 102206, P.R. China
}

Received March 12, 2012; Accepted May 14, 2012

DOI: 10.3892/ijmm.2012.1025

\begin{abstract}
Microtubule affinity-regulating kinase 4 (MARK4) belongs to a family of kinases that are able to actively phosphorylate the neuronal microtubule-associate proteins (MAPs), such as tau, MAP2 and the ubiquitous MAP4. Abnormal changes in tubulin and the profiles of tau have been previously reported in the human brain and animal transmissible spongiform encephalopathies (TSEs), which may be associated with abnormal alterations of various cellular kinases. To elucidate the possible role of MARK4 in TSE pathogenesis, the MARK4 levels in the brain tissues of scrapie-infected rodents and human prion diseases were evaluated using western blotting and immunohistochemical assays. The results revealed that at terminal stages of the diseases, MARK4 levels in the brain tissues of the scrapie 263K-infected hamsters, 139A-infected mice and a case of Creutzfeldt-Jakob disease (CJD, G114V gCJD) correlated with amounts of $\mathrm{PrP}^{\mathrm{Sc}}$ deposits that were almost undetectable. On the other hand MARK4 signals were noticeable in the brain tissues of a fatal familial insomnia (FFI) patient without $\mathrm{PrP}^{\mathrm{Sc}}$. The reduction of MARK4 was closely related to the prolonged incubation times. These results could be reproduced in SK-N-SH and PC12 cell lines after being exposed to the synthetic peptide PrP106-126. Accordingly, the levels
\end{abstract}

Correspondence to: Professor Xiao-Ping Dong, State Key Laboratory for Infectious Disease Prevention and Control, National Institute for Viral Disease Control and Prevention, Chinese Center for Disease Control and Prevention, Changbai Road 155, Beijing 102206, P.R. China

E-mail: dongxp238@sina.com

Dr Yong Liu, School of Medicine, Xi'an Jiao Tong University, Xi'an 710061, P.R. China

E-mail: liuy5599@mail.xjtu.edu.cn

Key words: transmissible spongiform encephalopathies, microtubule affinity-regulating kinase 4, prion, tau, Ser262, PrP106-126 of phosphorylated tau at Ser262 (p-tau262) in cultured cells exposed to PrP106-126, or the ratios of p-tau262/total tau in the brain tissues of $263 \mathrm{~K}$-infected hamsters were also significantly decreased. According to our data there is a correlation between a TSE pathological-associated decline of MARK4 in the brain tissues with the deposits of $\mathrm{PrP}^{\mathrm{Sc}}$. Reduction of MARK4 will result in abnormalities of tau phosphorylation, and possibly induce further detachment of microtubules and hinder microtubule transportation.

\section{Introduction}

Prion diseases, also called transmissible spongiform encephalopathies (TSEs), are a group of fatal neurodegenerative diseases characterized by neuronal loss and spongiform degeneration in the central nervous system (CNS). These diseases may affect many mammalian hosts, such as Creutzfeldt-Jakob disease (CJD), fatal familial insomnia (FFI), GerstmannStraussler-Scheinker (GSS) syndrome and Kuru in human, scrapie in sheep and goat, bovine spongiform encephalopathy (BSE) in cattle, and chronic wasting disease (CWD) in deer and elk $(1,2)$. It is commonly accepted that these type of diseases are caused by the conversion of a cellular protein $\mathrm{PrP}^{\mathrm{C}}$ in the CNS to an abnormally folded isoform, $\mathrm{PrP}^{\mathrm{Pc}}$, which is insoluble, proteinase K-resistant and infectious $(3,4)$. The accumulation of $\mathrm{PrP}^{\mathrm{Sc}}$ may be linked to the pathogenesis of prion diseases (5).

Microtubules and their associated proteins (MAPs) provide a dynamic network that is critical for cell mitosis, the establishment of cellular polarity and intracellular transport. These processes require a dynamic microtubule array and are regulated by motor proteins and structural MAPs, such as tau, MAP1B, MAP2 and MAP4. The binding of tau and other MAPs to microtubules are regulated instantaneously by phosphorylation on their tubulin binding repeats $(6,7)$. Our previous studies identified the profiles of tau and relevant phosphorylating kinases that are abnormally changed (8) and the levels of tubulin that are decreased in the brain tissues of experimental scrapie rodents and human CJD. Meanwhile, destruction of microtubule structures have been repeatedly 
observed in cells receiving genetic CJD (gCJD)-associated PrP mutants $(9,10)$. These data highlight that damage of microtubules is an essential process in the pathogenesis of TSEs.

Microtubule affinity-regulating kinase 4 (MARK4) belongs to a family of kinases that phosphorylate tau (p-tau) at the serine motif (11). It has two different splicing isoforms, MARK4-S, which is predominantly expressed in the brain, and MARK4-L, which is ubiquitously expressed in all tissues with high expression levels in testis, neural progenitors and glial tumors (12-14). In contrast to the other three MARK paralogs that all exhibit uniform cytoplasmic localization, MARK4 colocalizes with the centrosome and with microtubules in cultured cells. In differentiated neuroblastoma cells, MARK4 localizes prominently at the tips of the neurite-like processes (11). It has been reported that serine 262 in tau, which is specifically phosphorylated by MARK, is in a hyperphosphorylated form in the neurofibrillary deposits found in Alzheimer's disease brains (15). In addition, the microtubuledependent transport is regulated through MARK. In primary retinal ganglion cells, transfection with tau leads to the inhibition of axonal transport of mitochondria, amyloid precursor protein (APP) vesicles and other cell components. This leads to the starvation of axons and their vulnerability against stress. This transport inhibition can be rescued by phosphorylating tau with MARK (16). However, the alteration of the MARK family in TSEs remains unknown.

Our study of screening the transcriptional diversity in the brain tissues of human prion diseases with a commercial mRNA microarray shows that the expression of MARK4 is obviously decreased (unpublished data). In the present study, we present evidence that the MARK4 protein in the brain tissues of scrapie-agent $263 \mathrm{~K}$ and 139A-infected rodents is almost undetectable at the terminal stages, and the decline of MARK4 in the brain tissues of agent 263K-infected hamsters was closely related to the prolonged incubation time. MARK4 was extremely decreased in the brain regions with a mass of $\mathrm{PrP}^{\mathrm{Sc}}$ in a G114V gCJD patient, but was clearly observable in the regions with a minimum amount of $\operatorname{PrP}^{\mathrm{Sc}}$ or without detectable $\mathrm{PrP}^{\mathrm{Sc}}$ in a D178N FFI patient. Accordingly, the ratio of p-tau at Ser262 to total tau was also decreased in the brain tissues of agent $263 \mathrm{~K}$-infected hamsters. Those results could be reproduced in the cell lines exposed to the synthetic peptide PrP106-126, which possesses similar physicochemical and pathological properties to $\operatorname{PrP}^{\mathrm{Sc}}$. It indicates that the decrease of MARK4 in brain tissues may be involved in TSEs and may be correlated to the deposits of $\mathrm{PrP}^{\mathrm{Sc}}$.

\section{Materials and methods}

Ethics statement. Usage of the stored human and animal samples in this study was approved by the Ethics Committee of National Institute for Viral Disease Prevention and Control, China CDC. All signed informed consents were collected and stored by the China CJD Surveillance Center Housing. Experimental protocols were in accordance with the Chinese Regulations for the Administration of Affairs Concerning Experimental Animals.

Specimens. Four Chinese golden hamsters inoculated intracerebrally with hamster-adapted scrapie agent $263 \mathrm{~K}$ and 4 mice inoculated intracerebrally with mouse-adapted scrapie agent 139A were examined in this study. The incubation time of 263K-infected hamsters was 79.1 \pm 8.6 days (17), while that of 139A-infected mice was $153 \pm 4$ days (18). Brain samples of the hamsters infected with $263 \mathrm{~K}$ agent at the $20,40,60$ and 80 days after inoculation were collected. Samples of frontal lobe, parietal lobe, occipital lobe, temporal lobe, thalamus and callosal gyrus from a G114V gCJD patient $(19,20)$ and a D178N FFI patient (21) that were reported previously were also enrolled. All brains were removed surgically and stored at $-80^{\circ} \mathrm{C}$ until use. Brains from 4 normal hamsters and 4 normal mice were collected as controls.

Preparations of brain homogenates. The brain tissues $(10 \% \mathrm{w} / \mathrm{v})$ were homogenized in lysis buffer $(100 \mathrm{mM} \mathrm{NaCl}$, $10 \mathrm{mM}$ ethylenediaminetetraacetic acid, $0.5 \%$ Nonidet P-40, $0.5 \% \mathrm{Na}$ deoxycholate in $10 \mathrm{mM}$ Tris- $\mathrm{HCl}, \mathrm{pH}$ 7.4). The homogenates were centrifuged at 2,000 $\mathrm{xg}$ for $10 \mathrm{~min}$, and then the supernatants were collected and frozen at $-80^{\circ} \mathrm{C}$ for the further experiments.

Immunohistochemical (IHC) assays. The brain tissues were subjected to formalin fixation and paraffin embedding for conventional methodology (20). The slices were subjected to conventional immunostaining of MARK4, $\mathrm{PrP}^{\mathrm{Sc}}$ and GFAP. $\mathrm{PrP}^{\mathrm{Sc}}$ and GFAP stainings were performed with 1:250 diluted PrP specific monoclonal antibody (mAb; Dako, Denmark) 3F4 and 1:200 diluted GFAP polyclonal antibody (pAb; Santa Cruz Biotechnology, Inc., USA) according to the protocols described previously (17). For MARK4 staining, the slices were digested by enzymes for $30 \mathrm{sec}$ and $3 \%$ hydrogen peroxide-methanol for $10 \mathrm{~min}$. The slices were blocked with normal goat serum for $10 \mathrm{~min}$, then incubated with 1:100 diluted anti-MARK4 pAb (Abcam, UK) at $4^{\circ} \mathrm{C}$ overnight, and subsequently with horseradish peroxidase (HRP)-conjugated anti-rabbit IgG (Vector, USA) at $37^{\circ} \mathrm{C}$ for $1 \mathrm{~h}$. For visualization of immunostaining, a commercial DAB kit (Vector) was used, and the slices were counterstained with hematoxylin.

Western blot analyses. The brain tissue homogenates and cellular lysates were separated using $12 \%$ SDS-polyacrylamide gel electrophoresis (SDS-PAGE) and electronically transferred to nitrocellulose membranes. After blocking with 5\% non-fat milk powder in TBST (phosphate-buffered saline, pH 7.6, containing $0.05 \%$ Tween-20), the membranes were incubated with 1:1,000 diluted anti-MARK4 pAb, 1:5,000 diluted PrP specific mAb 3F4, 1:1,000 diluted anti-PrP mAb 1E4, 1:1,000-diluted anti-neuron specific enolase mAb (NSE; Abcam), 1:1,000 diluted anti-human $\beta$-actin mAb, 1:1,000 diluted anti-tau mAb tau13 (Santa Cruz Biotechnology, Inc.), or 1:1,000 diluted anti-p-tau at Ser262 pAb TAU [pS262] (Biosource, USA) at $4^{\circ} \mathrm{C}$ overnight. After washing thrice with TBST, the membranes were incubated with 1:8,000 diluted HRP-conjugated anti-rabbit or anti-mouse IgG (Boehringer, Germany) in TBST at room temperature for $1 \mathrm{~h}$, followed by detection of signals with an enhanced chemiluminescence detection kit (Amersham Pharmacia Biotech, USA).

Peptides. Peptides PrP106-126 (KTNMKHMAGAAAAG AVVGGLG) and scrambled (scr) peptide PrP106-126 (AVHT 
GLGAMAALNMVVGGAAGL) were synthesized and purified by Invitrogen (USA). Peptides were freshly dissolved in dimethyl sulfoxide (DMSO) to a concentration of $50 \mu \mathrm{M}$ before each experiment.

Cell culture. The adherent human neuroblastoma cell line SK-N-SH and rat pheochromocytoma cell line PC12 were cultured in Dulbecco's modified Eagle's medium (DMEM) supplemented with $10 \%$ fetal calf serum (Gibco, USA). Cells were maintained at $37^{\circ} \mathrm{C}$ in a humidified $5 \% \mathrm{CO}_{2}$ atmosphere.

Cell viability assays. SK-N-SH and PC12 cells at logarithmic growth stage were trypsinized and seeded on a 96-well plate at a concentration of $10^{4}$ cells/well before peptide treatments. Twelve hours after incubation with different concentrations of peptides, cell viability was determined using a commercially available Cell Counting kit (CCK-8; Dojindo, Japan). Briefly, $10 \mu \mathrm{l}$ of CCK-8 reagent were added to each well and incubated at $37^{\circ} \mathrm{C}$ for $1 \mathrm{~h}$ or until the media turned yellow. Absorbance was measured at $450 \mathrm{~nm}$ with a spectrophotometer. Each experiment was performed in triplicate and repeated at least three times.

Quantitative real-time PCR ( $q R T-P C R)$. Total-RNA from cells was extracted using the RNeasy mini kit (Qiagen, USA) reagent according to the manufacturer's instructions. Reverse transcription was performed with the Reverse Transcription System (Promega, USA). Briefly, $1 \mu$ l of each RT product was employed into subsequent PCR reactions. The primers for MARK4 were designed based on the sequences of human MARK4 in GenBank (NM_001199867.1) including MARK4(Hu), sense, 5'-GGCTATGAGGGTGAGGAGT TGAA-3' and antisense, 5'-GCGGTGGTAGGTGGAAG AGG-3'; $\beta$-actin, sense, 5'-CT ACAATGAGCTGCGTGTGGC-3' and antisense, 5'-CAGG TCCAGACGCAGGATGGC-3' ' qRT-PCR was performed on a 7900 Fast Real-Time PCR System (Applied Biosystems, USA) using the following conditions: $94^{\circ} \mathrm{C}$ for $15 \mathrm{sec}, 56^{\circ} \mathrm{C}$ for $40 \mathrm{sec}$, and $72^{\circ} \mathrm{C}$ for $30 \mathrm{sec}$ for 40 cycles. The expression level of mRNA was determined relative to that of the $\beta$-actin control. All real-time PCR reactions were performed in triplicate.

Statistical analyses. Statistical analyses were performed using the SPSS 17.0 statistical package. Quantitative analysis of immunoblot images was carried out using the ImageJ software. All data are presented as the mean $\pm \mathrm{SD}$. One-way ANOVA was used to assess the differences of OD450 values of CCK- 8 between treatment groups with mock group. p-values $<0.05$ were statistically significant.

\section{Results}

Decreases in MARK4 levels in the brain tissues of the scrapieinfected rodents at the terminal stages of the disease. To assess the potential changes in MARK4 levels in the brain tissues with TSEs, 4 hamsters infected with agent 263K (Ha-263K) and 4 mice infected with agent 139A (Mo-139A) were used in this study. The clinical features and the presence of $\mathrm{PrP}^{\mathrm{Sc}}$ in the brain tissues of the scrapie-infected animals have been previously reported $(8,22)$. The amounts of MARK4 and total PrP were evaluated with western blot analyses using individual antibodies. In accompaniment with large amounts of total PrP signals, almost no MARK4 signal was observed in the brain homogenates of two different kinds of scrapie-infected animals, whereas a clear MARK4 specific signal was observed in brain homogenates prepared from normal hamsters and mice at the same age (Fig. 1A).

To further investigate the effects of decreased MARK4 in scrapie-infected animals, the presence of MARK4 in the brain tissues of Ha-263K-infected animals were analyzed with IHC. Additionally, $\operatorname{PrP}^{\mathrm{Sc}}$ deposits and astrogliosis were monitored. As expected, large quantities of $\mathrm{PrP}^{\mathrm{Sc}}$ deposits were observed in the hippocampus and cortex of Ha-263K animals, which accompanied different sizes of vacuolation, but were not observed in the normal controls (Fig. 1B). More GFAP positively stained long and fibrous-like cells were detected in brain tissues of Ha-263K, while only small, filament-like structures appeared in wild-type animals (Fig. 1B). More round and granular MARK4 positively stained particles were monitored in the brain tissues of normal hamsters, but were almost unobservable in that of Ha-263K (Fig. 1B). The data strongly indicate that the levels of MARK4 in the brain tissues of scrapie experimental rodents are severely repressed at the terminal stages of diseases.

Decreases in MARK4 protein levels in the brain tissues of a G114V gCJD patient, but not in a FFI patient. To address the state of MARK4 in human prion diseases, the protein expression levels of MARK4 in six different brain regions, including the frontal lobe, parietal lobe, occipital lobe, temporal lobe, thalamus and callosal gyrus, from a G114V gCJD patient and a D178N FFI patient were assessed using western blotting. In accordance with the observations in scrapie-infected animals, in the six preparations from a G114V gCJD patient (Fig. 2A, left panel), there were almost no MARK4 signal observed in the four cortex regions. These regions were deposited with a mass of $\mathrm{PrP}^{\mathrm{Sc}}$, although weak, noticeable MARK4 bands in thalamus and callosal gyrus were deposited with a few $\operatorname{PrP}^{\mathrm{Sc}}$. Surprisingly, the preparations from a D178N FFI patient had nearly undetectable PrPres signals, while MARK4 specific bands were repeatedly observed in all tested regions (Fig. 2A, right panel). NSE-specific blots revealed similar signal intensities between the brain tissues of the two cases (Fig. 2A). The slides of the six brain regions from a G114V gCJD and a FFI patient were also screened with MARK4-specific IHC. In line with the results from our western blot analysis, clear, round and granular MARK4 positive-stained particles were observed in the brain regions of FFI, but not in the brain tissues of G114V gCJD (Fig. 2B). This result indicates that decreased MARK4 in brain tissues may be a common feature in TSEs, which is likely linked to the deposits of $\mathrm{PrP}^{\mathrm{Sc}}$.

Decreases of MARK4 are time-relative events correlated with the deposits of $\operatorname{PrP}^{S c}$. To ascertain possible dynamic alterations of MARK4 in the brain tissues of scrapie experimental hamsters during the incubation periods, brain samples infected with the agent Ha-263K at 20, 40, 60 and 80 days post-inoculation (dpi) were collected. PrP specific western blot analysis identified PK-resistant PrP signals (PrPres) in 40 dpi preparations, but not in control and 20 dpi (Fig. 3A). The signal intensities of PrPres became more intense with 
A

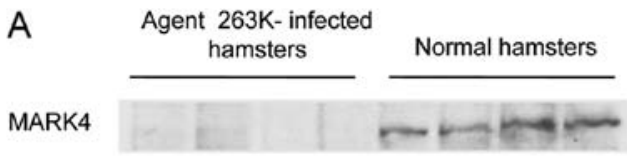

PrP (PK-)

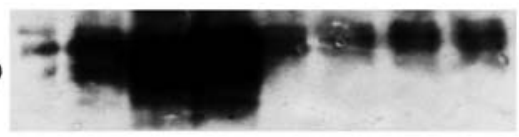

$\beta$-actin

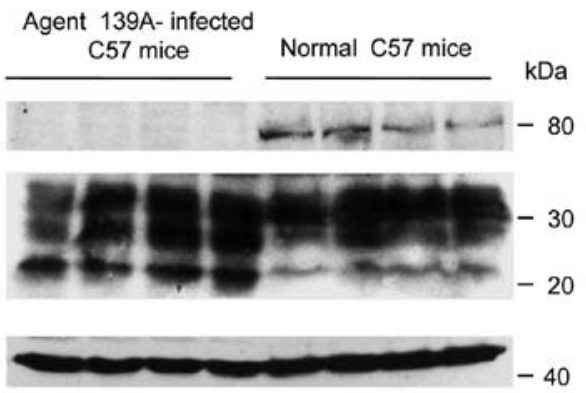

Normal hamsters

B

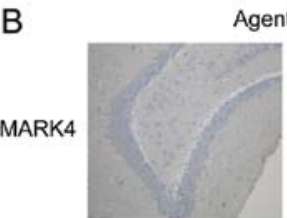

Agent 263K- infected hamsters

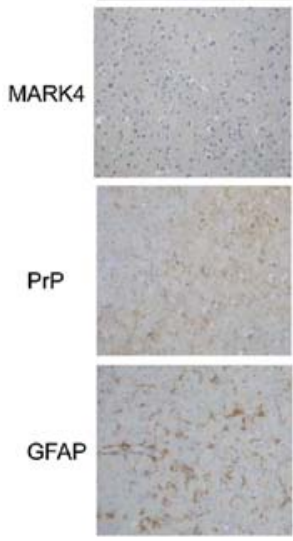

$\times 100$
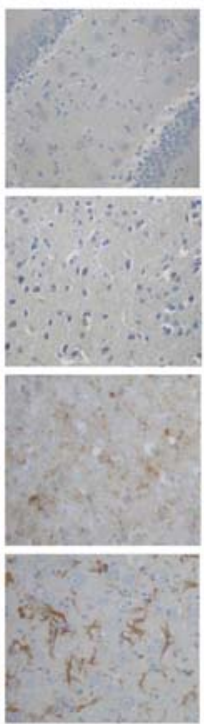

$\times 200$

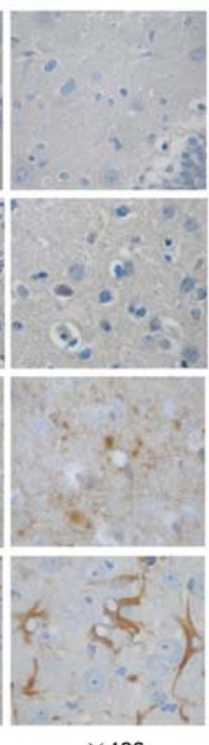

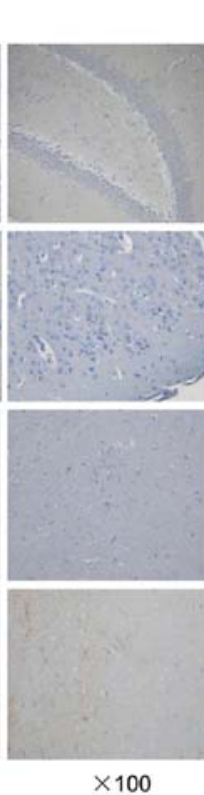

$\times 100$

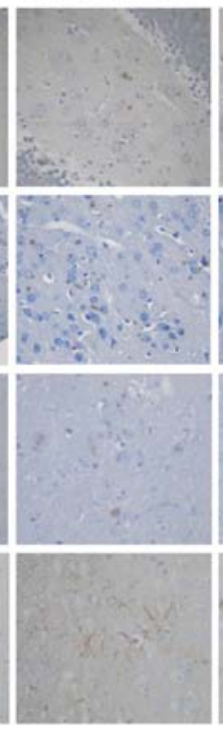

$\times 200$

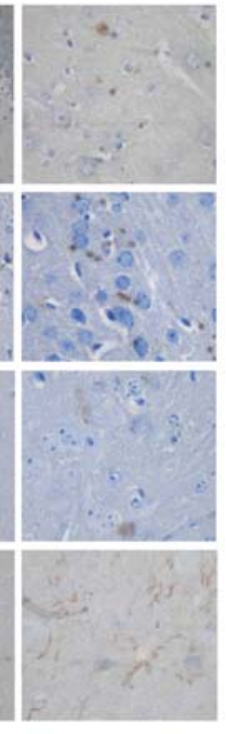

$\times 400$

Figure 1. Analysis of the levels of MARK4 in the brain tissues of scrapie-infected rodents. (A) Western blot analyses of brain tissues of scrapie agent $263 \mathrm{~K}$-infected hamsters (left) and 139A-infected mice (right). The immunoblots of MARK4, total PrP and $\beta$-actin as an internal control are shown in upper, middle and lower parts, respectively. (B) Immunohistochemistry assays of MARK4, total PrP and GFAP in the brain of a normal and a scrapie agent 263K-infected hamster. The first row shows MARK4 in the hippocampus, and the second to fourth rows show MARK4, total PrP, and GFAP in the cortex, respectively. Magnifications are shown at the bottom.

prolonged incubation, showing a time dependency for this signal. In contrast, MARK4 was clearly detectable in the brain tissues of normal hamsters, which significantly weakened in samples infected with $\mathrm{Ha}-263 \mathrm{~K}$ at $20 \mathrm{dpi}$, and continually decreased with time, eventually disappearing in the Ha-263K sample at 80 dpi (Fig. 3A). Quantitative analyses of the gray values of $\mathrm{PrP}^{\mathrm{Sc}}$ and MARK4 of each sample, normalized to the individual values of $\beta$-actin, revealed two opposite fluctuating curves, the increasing curve of $\mathrm{PrP}^{\mathrm{Sc}}$ and the declining curve of MARK4, along with the incubation period (Fig. 3B). These results illustrate that the levels of MARK4 in the brains of the scrapie-infected animals decline with prolonged incubation, which correlates with the increase of $\mathrm{PrP}^{\mathrm{Sc}}$.

Exposure to peptide PrP106-126 reduces the levels of MARK4 in cultured cells. Peptide PrP106-126 shows cytotoxicity in several cultured cells in vitro, which may partially mimic the features of $\operatorname{PrP}^{\mathrm{Sc}}$ (23-25). To assess the possible changes in cellular MARK4 following cytotoxicity induced by exposure to peptide PrP106-126, neuroblastoma cell line SK-N-SH and rat pheochromocytoma cell line PC12 were exposed to different concentrations of PrP106-126. Both cell lines showed distinct morphological changes and low cell viability when exposed to PrP106-126, but did not show obvious changes when exposed to DMSO or the same amount of scramble PrP106-126 peptide (Fig. 4A and B). After treatment with PrP peptides for 6 and $12 \mathrm{~h}$, the levels of MARK4 were evaluated using western blot analyses. Two MARK4-specific bands in both cell lysates migrate near the positions of 85 and $80 \mathrm{kDa}$ (Fig. 4C), representing large and small fragments of MARK4 (MARK4-L and MARK4-S), respectively. The protein level of MARK4-L and MARK4-S both decreased after PrP106-126 treatment compared with scramble peptide or DMSO treatment. Quantitative analyses of the relative gray numerical values normalized to that of $\beta$-actin revealed that the levels of MARK4-L and MARK4-S in the cells treated with PrP106-126 were significantly lower than the mock-treated cells, whereas the cells exposed to DMSO and scrambled PrP peptide were slightly decreased without significant difference (Fig. 4D). The reductions of MARK4-S after treatment of PrP106-126 were even more remarkable. Prolonging the treatment times of PrP106-126 resulted in significant reductions of MARK4 in both cell lines.

To address the expression profile of MARK4 in the cells after challenging with PrP106-126, a qRT-PCR specific for MARK4 was performed. RT-PCR assays using extracted RNA 
A
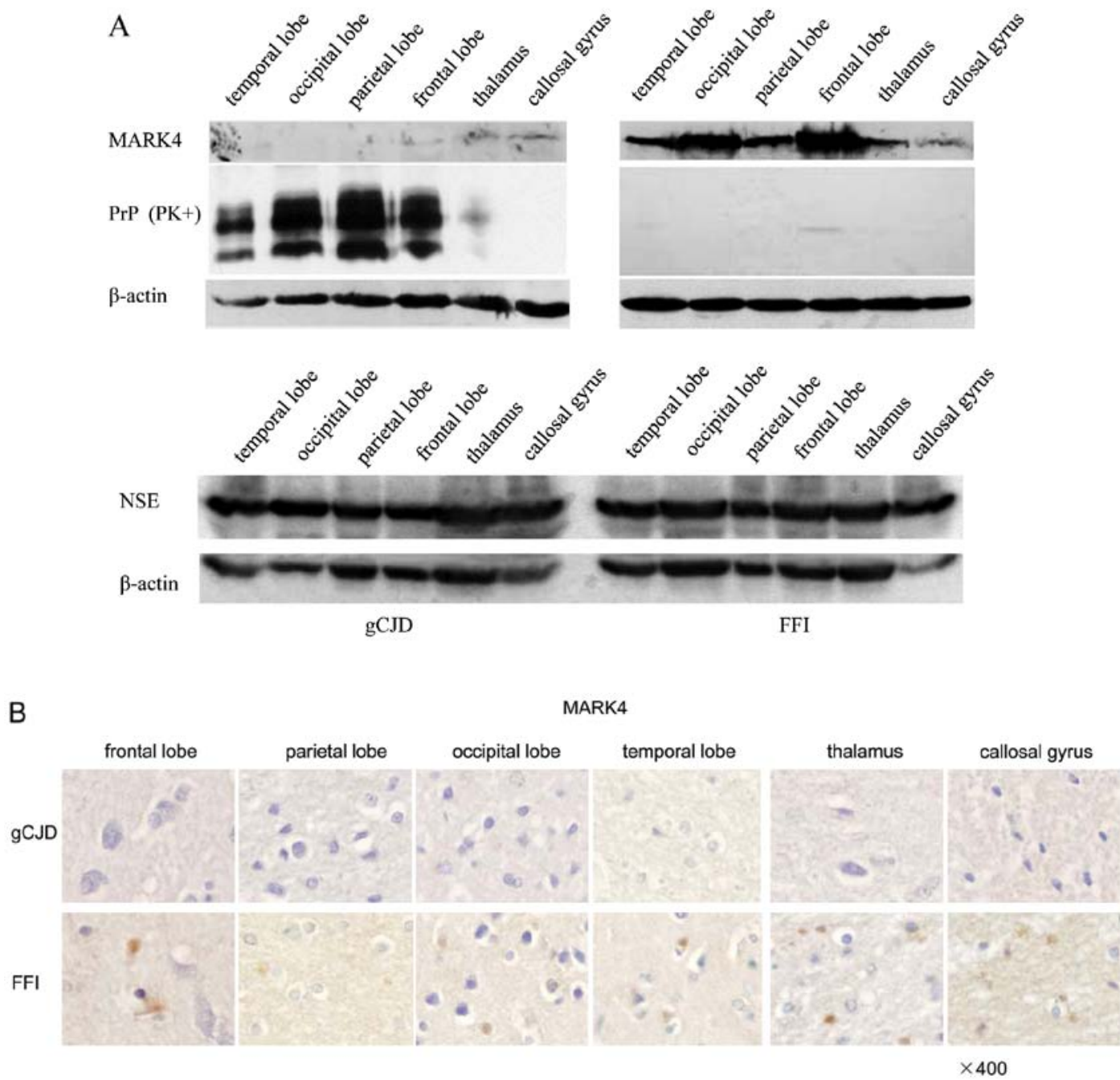

Figure 2. Analysis of the levels of MARK4 in the brain tissues of human prion diseases. (A) Western blot analyses of six brain regions of a G114V gCJD patient (left panel) and a D178N FFI patient (right panel). Immunoblots for MARK4, $\operatorname{PrP}^{\text {Sc }}$ (digested with $20 \mu \mathrm{g} / \mathrm{ml} \mathrm{PK}$ ), NSE and $\beta$-actin, internal control, are indicated on the left, respectively. The brain regions are indicated at the top. (B) IHC assays of MARK4. The brain slides from G114V gCJD and FFI are indicated on the left. Magnifications are shown at the bottom.

A

Normal hamsters Agent 263K- infected hamsters

\section{$20 \mathrm{~d} 20 \mathrm{~d} 40 \mathrm{~d} 40 \mathrm{~d} 60 \mathrm{~d} 60 \mathrm{~d} 80 \mathrm{~d} \mathrm{kDa}$}

MARK4

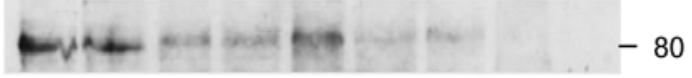

$\beta$-actin

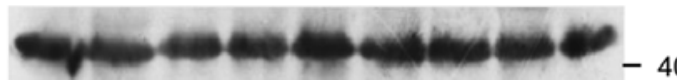

Normal hamsters Agent 263K- infected hamsters

$20 \mathrm{~d} 20 \mathrm{~d} 40 \mathrm{~d} 40 \mathrm{~d} 60 \mathrm{~d} 60 \mathrm{~d} 80 \mathrm{~d} \quad \mathrm{kDa}$

$\operatorname{PrP}$

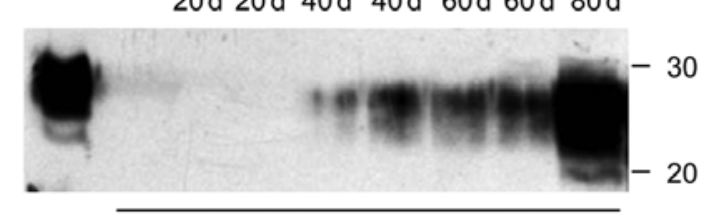

PK-
B

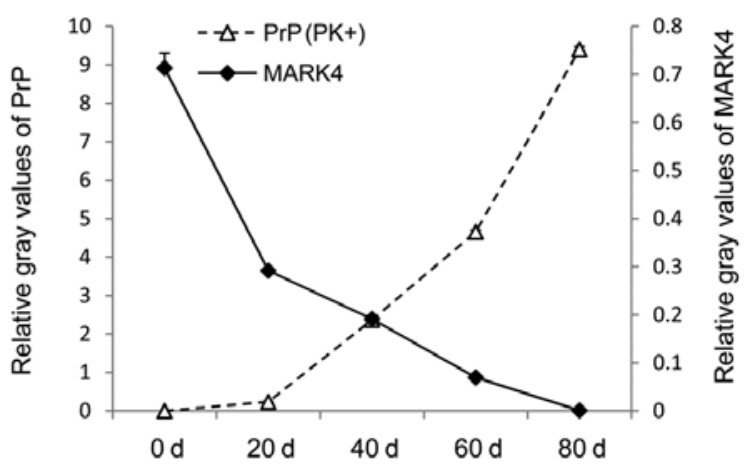

Figure 3. Dynamic analysis of MARK4 and $\mathrm{PrP}^{\mathrm{Sc}}$ in the brain tissues of normal and scrapie agent 263K-infected hamsters during the incubation period. (A) Western blot analyses of MARK4, $\beta$-actin and $\mathrm{PrP}^{\mathrm{Sc}}$ (digested with $50 \mu \mathrm{g} / \mathrm{ml} \mathrm{PK}$ ) from Ha-263K and normal controls are shown on the left. The sampling times post-inoculation are shown as days (d) at the top. (B) Quantitative analyses of each gray numerical value of PrP ${ }^{\mathrm{Sc}}$ and of MARK4 vs. individual one of $\beta$-actin. The average values were calculated from two infected hamsters or two normal hamsters. Data are representative of three independent experiments. 
A

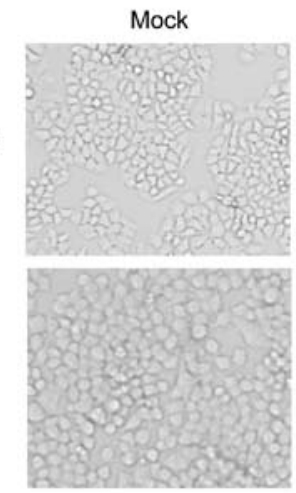

DMSO

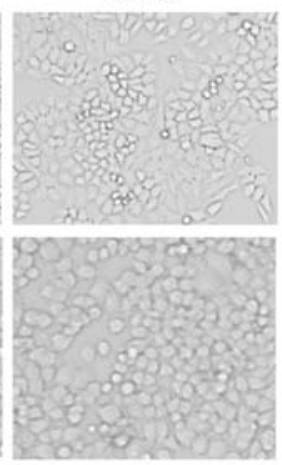

B

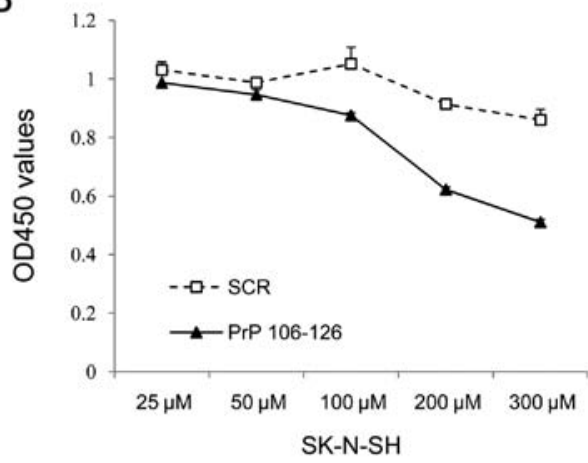

C
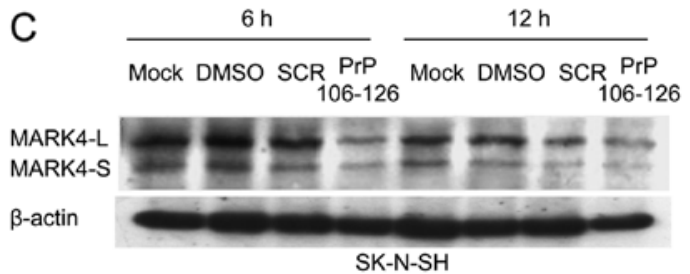

SK-N-SH
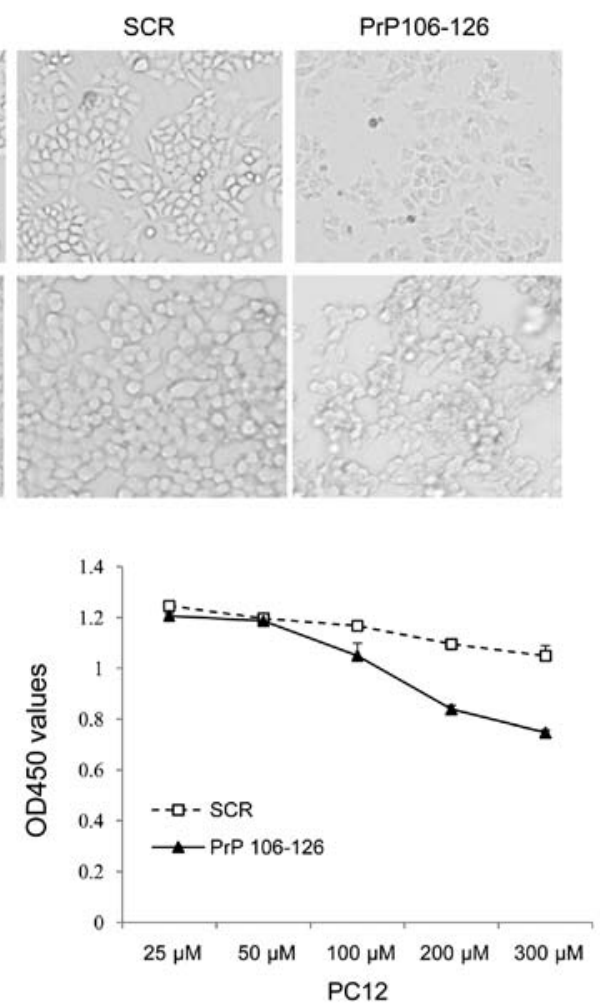

$6 \mathrm{~h}$

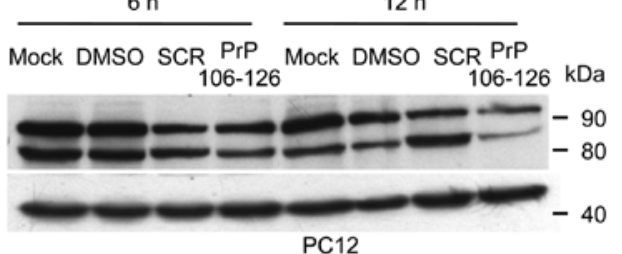

D
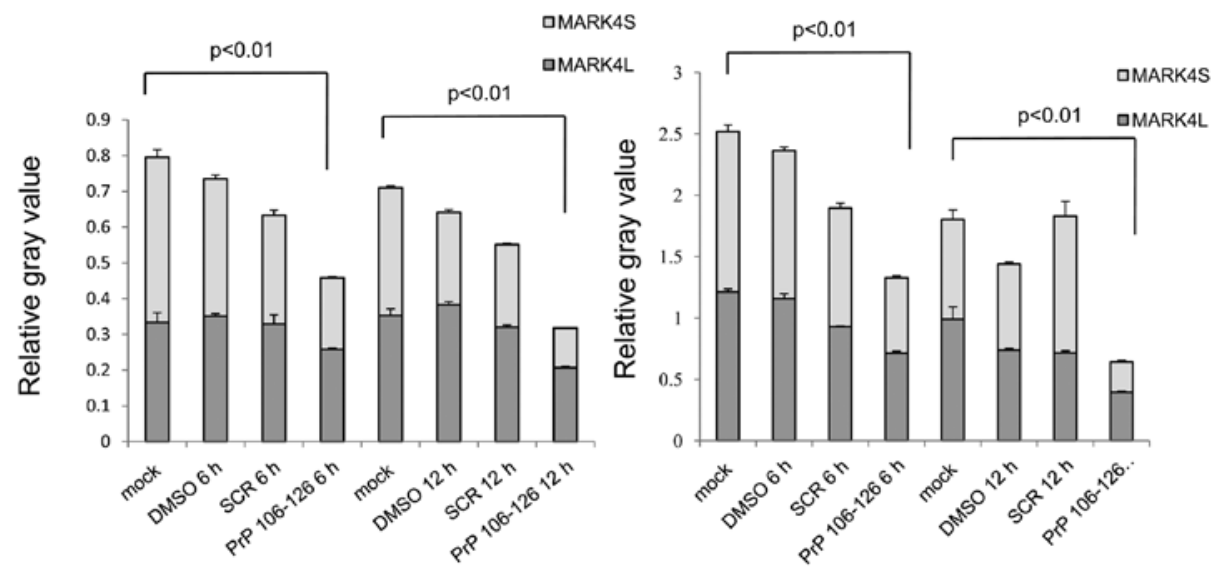

Figure 4. Analysis of the MARK4 levels in SK-N-SH and PC12 cells exposed to synthetic peptide PrP106-126. (A) The images of the morphological cytotoxicity $12 \mathrm{~h}$ after treatments of PrP106-126, scrambled peptide PrP106-126 (SCR) or DMSO. (B) The cell viabilities of SK-N-SH and PC12 cells $12 \mathrm{~h}$ after exposure to different concentrations of PrP106-126 or SCR. The average data of each preparation were calculated based on three independent experiments and represented as the mean \pm SD. (C) Western blot analyses of the SK-N-SH and PC12 cell lysates after 6 and 12 h exposure to PrP106-126, SCR or DMSO. The immunoblots of MARK4 and $\beta$-actin in the cells lysates are indicated on the left. (D) Quantitative analyses of each gray numerical value of MARK4 vs. that of $\beta$-actin. The average relative gray value is calculated from three independent blots and presented as the mean \pm SD.

from the cells exposed to PrP106-126 for 6 and $12 \mathrm{~h}$ as well as the cells receiving DMSO or scramble PrP peptide for $12 \mathrm{~h}$ revealed a 325-bp amplified fragment after using RNA isolated from cells which were further verified to be MARK4-specific sequences (data not shown). Real-time PCR for MARK4 demonstrated that the amounts of MARK4 transcripts in the cells exposed to PrP106-126 were profoundly downregulated when compared with that of the controls, showing a statistically significant difference both in the SK-N-SH and PC12 cell lines (Fig. 5 and Table I). Meanwhile, treatment with PrP106- 

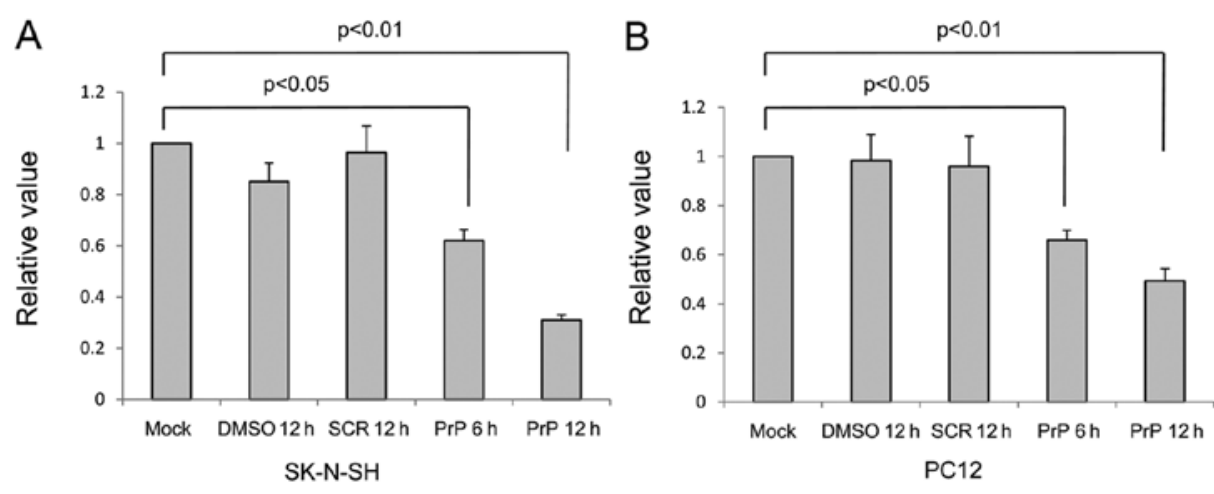

Figure 5. Real-time PCR analyses of the mRNA transcript of MARK4 in two different cell lines exposed to the PrP106-126 peptide. The mRNA transcriptional levels of MARK4 of (A) SK-N-SH cells and (B) PC12 cells are normalized to that of $\beta$-actin. For each gene, the relative intensity from treated cells is relative to that gene from mock, which is set to 1 . Data are representative of three independent experiments.

Table I. Comparison of the amplification data of MARK4-specific mRNAs in various cell preparations by real-time PCR.

\begin{tabular}{lccccc}
\hline & $\begin{array}{c}\text { MARK4 } \\
\text { Average } \mathrm{C}_{\mathrm{t}}\end{array}$ & $\begin{array}{c}\beta \text {-actin } \\
\text { Average } \mathrm{C}_{\mathrm{t}}\end{array}$ & Average $\Delta \mathrm{C}_{\mathrm{t}}^{\mathrm{a}}$ & $\Delta \Delta \mathrm{C}_{\mathrm{t}}^{\mathrm{b}}$ & $2^{-\Delta \Delta \mathrm{Ct}}$ \\
\hline PC-12 & & & & \\
Mock & 23.74 & 13.84 & 9.90 & 0.00 & $1.00 \pm 0.00$ \\
DMSO & 23.67 & 13.53 & 10.14 & 0.24 & $0.85 \pm 0.07$ \\
SCR & 23.76 & 13.82 & 9.94 & 0.04 & $0.97 \pm 0.11$ \\
PrP 6 h & 23.59 & 12.98 & 10.61 & 0.71 & $0.61 \pm 0.04$ \\
PrP 12 h & 25.01 & 13.36 & 11.65 & 1.75 & $0.30 \pm 0.02$ \\
SK-N-SH & & & & & \\
Mock & 22.56 & 13.10 & 9.46 & 0.00 & $1.00 \pm 0.00$ \\
DMSO & 22.76 & 13.11 & 9.65 & 0.06 & $0.97 \pm 0.11$ \\
SCR & 22.85 & 13.20 & 10.02 & 0.60 & $0.66 \pm 0.12$ \\
PrP 6 h & 23.64 & 13.62 & 10.81 & 1.01 & $0.49 \pm 0.04$ \\
PrP 12 h & 24.01 & 13.20 & & & 0.05 \\
\hline
\end{tabular}

${ }^{a}$ MARK4 $\Delta \mathrm{C}_{\mathrm{t}}$ is the $\mathrm{C}_{\mathrm{t}}$ for the target gene normalized to an endogenous control $(\beta$-actin $)\left(\mathrm{C}_{\mathrm{t} \text { MARK4 }}-\mathrm{C}_{\mathrm{t} \beta \text {-actin }}\right)$. ${ }^{\mathrm{b}}$ The calculation of $\Delta \Delta \mathrm{C}_{\mathrm{t}}$ involves subtraction by the $\Delta \mathrm{C}_{\mathrm{t}}$ calibrator value (MARK4) $\left(\Delta \mathrm{C}_{\mathrm{t}}-\Delta \mathrm{C}_{\mathrm{t} \text { mock }}\right)$.

126 for $12 \mathrm{~h}$ decreased the MARK4 expression in cells more than the $6 \mathrm{~h}$ treatment. These data suggest that PrP106-126 downregulates the expression of the endogenous MARK4 when it is cytotoxic to cultured cells.

Phosphorylated tau at serine 262 is downregulated in the PrP106-126-treated cells and in scrapie-infected hamster brains. Tau is a group of molecular mass proteins of 45-66 kDa with multiple phosphorylation sites. To test whether p-tau262, which is considered a substrate of MARK4 (26), was influenced as MARK4 decreased, the lysates of PC12 and SK-N-SH cells treated with PrP106-126 were tested using western blot analyses with a mAb for total tau (tau13) and a pAb specific for p-tau at Ser262 [TAU (pS262)]. In SK-N-SH cells, several bands were detected after staining with $\mathrm{mAb}$ tau13 and pAb TAU (pS262). Among them, only the signal of $\mathrm{p}$-tau262 in the cells after exposure of PrP106-126 for $12 \mathrm{~h}$ was markedly weak (Fig. 6A). In PC12 cells, one signal band was observed after immunoblotting with mAb tau13 and pAb TAU
(pS262). Similarly, the signal of p-tau262 in the cells exposed to PrP106-126 for $12 \mathrm{~h}$ was significantly weaker compared to the other cells (Fig. 6A). The relative gray values of the signals of total tau and p-tau 262 from each reaction were normalized to $\beta$-actin. Analyses of the ratios of the digital data of $p$-tau 262 to that of total tau revealed significantly lower values $(\mathrm{p}<0.05)$ in the preparations treated with PrP106-126 for $12 \mathrm{~h}$, both in SK-N-SH and PC-12 cells (Fig. 6B). These finding highlight that treatment of PrP106-126 in cultured cells does not alter the level of total tau, but induces a reduction of p-tau262.

Four brain homogenates from either Ha-263K or normal hamsters were also analyzed using western blot analyses with the antibodies against tau and p-tau262. In line with our previous data (8), the levels of total tau increased in the brain tissues of Ha-263K, showing a statistically significant difference in the signal intensity compared with that of normal controls (p<0.01) (Fig. 7B). The levels of p-tau262 in Ha-263K also increased but were not statistically significant between the two groups ( $>0.05$ ) (Fig. 7B). Calculations of the ratios 
A
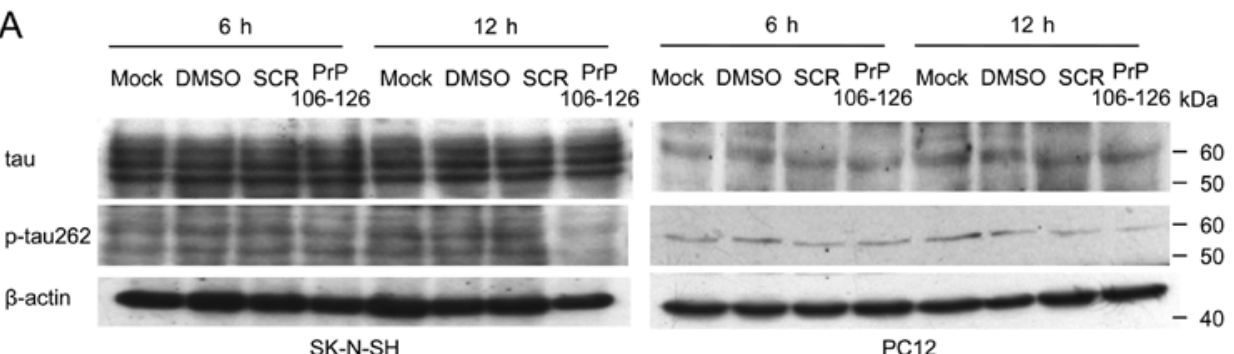

B p-tau 262tau

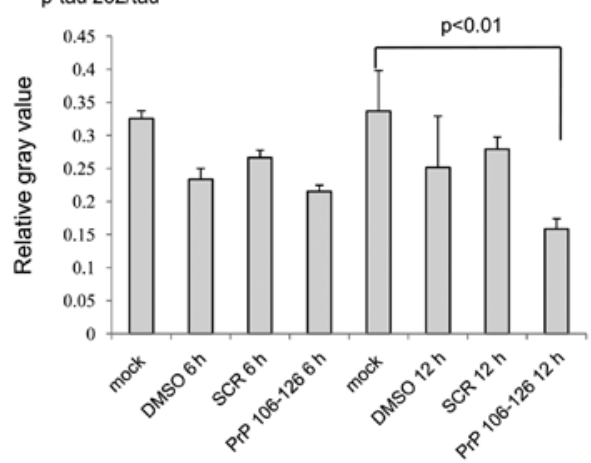

p-tau 262/tau

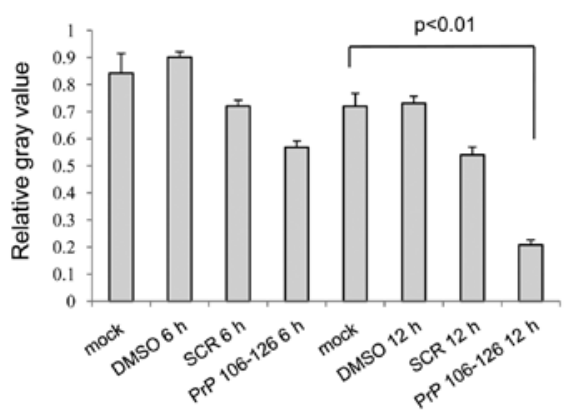

Figure 6. Analysis of the levels of total tau and p-tau262 in SK-N-SH and PC12 cells exposed to synthetic peptide PrP106-126. (A) Western blot analyses of the cultured cells 6 and $12 \mathrm{~h}$ after exposure to PrP106-126, SCR or DMSO. The immunoblots of tau, p-tau262 and $\beta$-actin on the cell lysates are shown. (B) Quantitative analysis of each gray numerical value of total tau or p-tau262 vs. that of $\beta$-actin. The average relative gray value is calculated from three independent blots. The ratios of p-tau262 to total tau are presented on the $y$-axis and presented as the mean \pm SD.

A

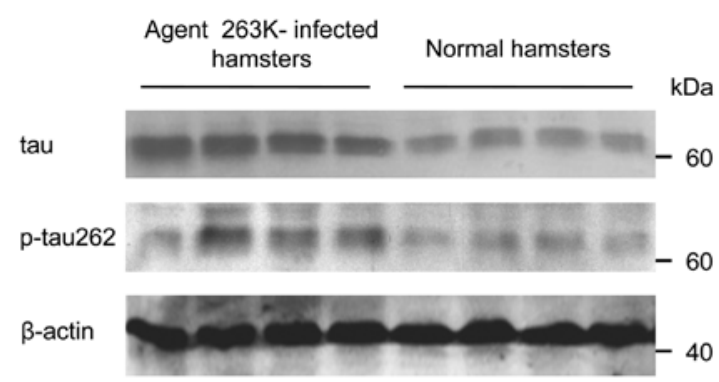

B
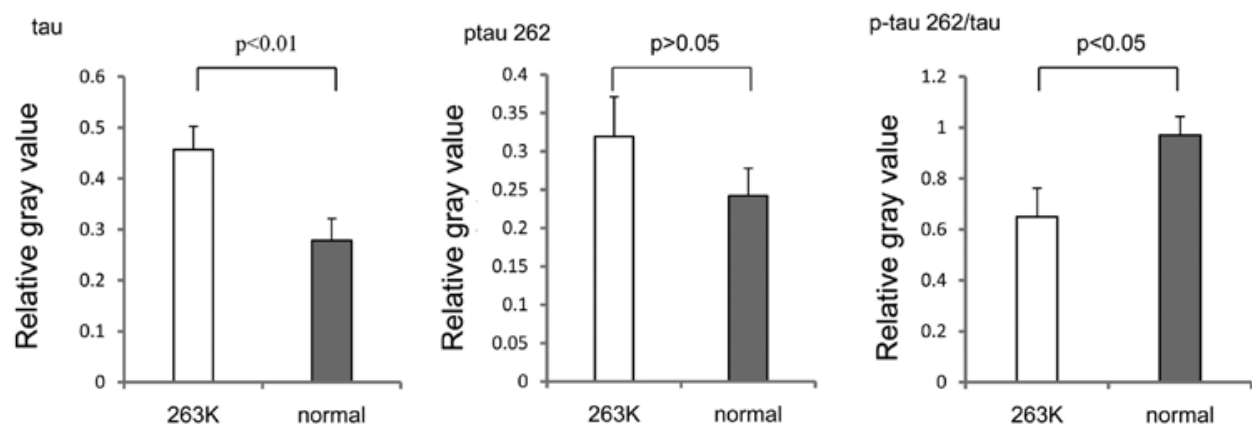

Figure 7. Analysis of the levels of total tau and p-tau262 in the brain tissues of scrapie agent 263K-infected hamsters. (A) Western blot analyses for total tau, p-tau262 and $\beta$-actin are shown in upper, middle and lower parts. (B) Quantitative analysis of the average gray numerical values of total tau (left), p-tau262 (middle), and the ratio of p-tau262/total tau (right) between infected and normal hamsters. The average gray values were calculated from 4 infected hamsters or 4 normal hamsters after being normalized to $\beta$-actin and are presented as the mean \pm SD.

of p-tau262 to total tau identified that the average value in Ha-263K was clearly lower than that of the normal control $(\mathrm{p}<0.05)$ (Fig. 7B). This result may suggest that although the amounts of total tau in scrapie-infected rodents increased at the terminal stage of the disease, the portion of p-tau262 decreased.

\section{Discussion}

A comprehensive sequence analysis of the human genome (27) revealed that the human MARK gene family consists of four paralogous genes. MARK4 shares 75\% homology with MARK1-3 and is predominantly expressed in the brain (11). 
In differentiated neuroblastoma cells, MARK4 is localized predominantly at the tips of neurite-like processes and a tandem affinity-purified MARK4 protein complex contains $\alpha-, \beta$ - and $\gamma$-tubulin (11). MARK4 is present in a number of neuronal populations and MARK4-S is upregulated in human neural progenitor cells (HNPCs) and NTera2 (NT2) cells from the very early stages of neuronal differentiation, suggesting that MARK4 is a neuron-specific marker in the CNS (28). Upregulation of MARK4-S during neuronal differentiation indicates that it may play a specialized role in neurons.

In our ongoing study on the global gene expression profiles in the brain tissues of a G114V gCJD patient, we found that the transcriptional levels of MARK4 are significantly lower than those of the control (unpublished data). In this study, we first used both western blot analysis and IHC assays to show that the MARK4 expression levels are disrupted in the brain tissues of two different scrapie-infected experimental rodents at the terminal stages of their diseases. Similarly, MARK4 was hardly detectable in various brain regions of the G114V gCJD human sample. These phenomena correlate well with the pathological features of most human and animal TSEs, representing severe neuron loss.

Our dynamic assays conducted on the brain tissues of a scrapie $263 \mathrm{~K}$ experimental hamster, collected over several incubation periods, showed a clear opposing relationship between the increase of $\mathrm{PrP}^{\mathrm{Sc}}$ and the decrease of MARK4. Additionally, the reduction of MARK4 in brain tissues seems to appear earlier than the presence of detectable $\mathrm{PrP}^{\mathrm{Sc}}$ in this model. Such phenomena appear to be verified by the observations in further assays with two different genetic human prion diseases, in which almost no MARK4 was detectable in the brain tissues of a G114V gCJD case with $\mathrm{PrP}^{\mathrm{Sc}}$, but MARK4 could be detected in the brain tissues of a FFI case without detectable $\mathrm{PrP}^{\mathrm{Sc}}$. This highlights that the disruption of MARK4 expression in TSEs closely accompanies the deposits of $\mathrm{PrP}^{\mathrm{Sc}}$, which possibly reflects the different situations of neuron loss among various TSE subtypes.

NSE is a widely used biomarker for neurons. Although neuronal loss is morphologically observed, due to the lack of normal human brain tissues, we cannot address the exact status of neuron damage in CNS of the G114V gCJD and FFI cases at the terminal stages. However, NSE-specific signals are repeatedly detected in the brain tissues of G114V gCJD and FFI cases using western blot analysis and IHC assays (unpublished data). The intensities of the NSE signals in these two cases differ slightly in this study, highlighting a possibility that MARK4positive neurons are more susceptible and more affected. Astrogliosis is another hallmark in most human and animal TSEs. Our previous studies on these two human genetic prion diseases have revealed abundant gliosis with GFAP-specific IHC assays $(20,21)$. It appears that the astrogliosis in the brain tissues have little influence on the MARK4 expression.

The influence of $\mathrm{PrP}^{\mathrm{Sc}}$ on the MARK4 expression partially reappears in cultured cells exposed to the synthetic peptide PrP106-126, which is believed to retain the ability of $\mathrm{PrP}^{\mathrm{Sc}}$ to aggregate into amyloid-like fibrils and the tendency to adopt a mostly $\beta$-sheet structure $(29,30)$. Along with the cytotoxicity of PrP106-126, the cellular MARK4 levels drop remarkably. Not surprisingly, cultured cell lines used in this study possess two different lengths of MARK4, MARK4-L and MARK4-S, which are different from the MARK4 profile observed in brain tissues. These results are in accordance with the distribution of two different MARK4 splicing isoforms. MARK4-L protein is highly expressed in cancers, such as hepatocarcinoma, Kato et al (12) and glioma cell lines (14,31), but infrequently in brains. Two cell lines used in our study are both cancerderived cells. Moreover, although PrP106-126 is able to downregulate the levels of both MARK4 isoforms, it elicits a stronger reduction for the MARK4-S isoform. As a kinase, MARK4 mediates the phosphorylation on tau at Ser262. Our data illustrate that PrP106-126 reduces the cellular MARK4 levels and subsequently reduces the amounts of p-tau262, but does not change the levels of cellular total tau. Meanwhile the ratios of p-tau262/total tau in the brain tissues of scrapieinfected hamsters show downregulation, although the absolute amounts of p-tau262 and total tau are increased. These data suggest a positive correlation between MARK4 and p-tau262.

Previously, we discoverd that the tau profile in the brain tissues of hamsters infected with scrapie strains $263 \mathrm{~K}$ or $139 \mathrm{~A}$ show particular changes that are possibly associated with the alteration of phosphate kinases, in which p-tau at Ser202/ Thr205 increased and p-tau at Ser396 and Ser404 decreased, accompanied by increased CDK5 and reduced GSK3 $\beta$ levels (8). Hyperphosphorylated tau, which can aggregate into typical paired helical filaments (PHFs), is the main component of the neurofibrillary tangles found in the brains of patients with Alzheimer's disease $(32,33)$. However, the relationship between toxicity and phosphorylation of tau has not been clearly illustrated. Microtubule binding activity may play a crucial role in the regulation of tau toxicity. Abnormality in tau phosphorylation will induce instability or detachment of the cytoskeleton.

The reason why the MARK4 expression is decreased in the terminal stages of prion diseases remains unclear. One possibility may be due to the mistaken degradation of MARK4. MARK4 belongs to the AMP-activated protein kinase (AMPK) family, which functions as a metabolic fuel gauge that is activated in response to environmental stressors needed to restore the cellular energy balance (34). The AMPK-related kinases are polyubiquitinated in vivo (35). The polyubiquitination of proteins is the triggering signal that leads to the degradation of the protein in the proteasome. It has been reported that MARK4 works as a partner, showing significant interactions with the human $\operatorname{PrP}^{\mathrm{C}}$ interactome in a high-density microarray analysis (36). One may presume that during TSE progression, MARK4 interacts with $\mathrm{PrP}^{\mathrm{Sc}}$ and triggers degradation by the proteasome.

It has been reported that tau inhibits kinesin-dependent transport of peroxisomes, neurofilaments, and Golgi-derived vesicles into neurites. In particular, tau inhibits the transport of an amyloid precursor protein into axons and dendrites, causing its accumulation in the cell body (37). The expression of tau protein in $\mathrm{CHO}$ cells reveals that both of the microtubule motors, kinesin and dynein, are inhibited (38). However, this inhibition can be regulated by MARK. Expression levels of MARK causes the phosphorylation of MAPs at their KXGS motifs, thereby detaching MAPs from the microtubules and facilitating the transport of particles (16). Hence, the repressed level of MARK4 in the brains of prion diseases may block the transportation through microtubules and accelerate disease progression. 


\section{Acknowledgements}

This study was supported by the China Mega-Project for Infectious Disease (2009ZX10004-101, 2008ZX10004-008), the National Basic Research Program of China (973 Program) (2007CB310505), the Chinese National Natural Science Foundation grant (30800975) and the SKLID Development grant (2008SKLID102, 2011SKLID204 and 2011SKLID211).

\section{References}

1. Prusiner SB: Prions. Proc Natl Acad Sci USA 95: 13363-13383, 1998.

2. Caughey B and Chesebro B: Prion protein and the transmissible spongiform encephalopathies. Trends Cell Biol 7: 56-62, 1997.

3. Castilla J, Saa P, Hetz C and Soto C: In vitro generation of infectious scrapie prions. Cell 121: 195-206, 2005.

4. Legname G, Baskakov IV, Nguyen HO, et al: Synthetic mammalian prions. Science 305: 673-676, 2004.

5. McKinley MP, Bolton DC and Prusiner SB: A protease-resistant protein is a structural component of the scrapie prion. Cell 35 57-62, 1983.

6. Desai A and Mitchison TJ: Microtubule polymerization dynamics. Annu Rev Cell Dev Biol 13: 83-117, 1997.

7. Gundersen GG: Evolutionary conservation of microtubulecapture mechanisms. Nat Rev Mol Cell Biol 3: 296-304, 2002.

8. Wang GR, Shi S, Gao C, et al: Changes of tau profiles in brains of the hamsters infected with scrapie strains $263 \mathrm{~K}$ or $139 \mathrm{~A}$ possibly associated with the alteration of phosphate kinases. BMC Infect Dis 10: 86, 2010.

9. Dong CF, Shi S, Wang XF, et al: The N-terminus of PrP is responsible for interacting with tubulin and fCJD related PrP mutants possess stronger inhibitive effect on microtubule assembly in vitro. Arch Biochem Biophys 470: 83-92, 2008.

10. Li XL, Wang GR, Jing YY, et al: Cytosolic PrP induces apoptosis of cell by disrupting microtubule assembly. J Mol Neurosci 43: $316-325,2011$

11. Trinczek B, Brajenovic M, Ebneth A and Drewes G: MARK4 is a novel microtubule-associated proteins/microtubule affinityregulating kinase that binds to the cellular microtubule network and to centrosomes. J Biol Chem 279: 5915-5923, 2004.

12. Kato T, Satoh S, Okabe H, et al: Isolation of a novel human gene, MARKL1, homologous to MARK3 and its involvement in hepatocellular carcinogenesis. Neoplasia 3: 4-9, 2001.

13. Schneider A, Laage R, von Ahsen O, et al: Identification of regulated genes during permanent focal cerebral ischaemia: characterization of the protein kinase 9b5/MARKL1/MARK4. J Neurochem 88: 1114-1126, 2004.

14. Beghini A, Magnani I, Roversi G, et al: The neural progenitorrestricted isoform of the MARK4 gene in 19q13.2 is upregulated in human gliomas and overexpressed in a subset of glioblastoma cell lines. Oncogene 22: 2581-2591, 2003.

15. Hasegawa M, Morishima-Kawashima M, Takio K, Suzuki M, Titani $\mathrm{K}$ and Ihara Y: Protein sequence and mass spectrometric analyses of tau in the Alzheimer's disease brain. J Biol Chem 267: 17047-17054, 1992.

16. Mandelkow EM, Thies E, Trinczek B, Biernat J and Mandelkow E: MARK/PAR1 kinase is a regulator of microtubule-dependent transport in axons. J Cell Biol 167: 99-110, 2004.

17. Zhang J, Chen L, Zhang BY, et al: Comparison study on clinical and neuropathological characteristics of hamsters inoculated with scrapie strain $263 \mathrm{~K}$ in different challenging pathways. Biomed Environ Sci 17: 65-78, 2004.

18. Kim YS, Carp RI, Callahan SM and Wisniewski HM: Incubation periods and survival times for mice injected stereotaxically with three scrapie strains in different brain regions. J Gen Virol 68: 695-702, 1987.
19. Ye J, Han J, Shi Q, et al: Human prion disease with a G114V mutation and epidemiological studies in a Chinese family: a case series. J Med Case Rep 2: 331, 2008.

20. Shi Q, Zhang BY, Gao C, Han J, Wang GR, Chen C, Tian C and Dong XP: The pathological and pathogenic characteristics in various brain regions from a Chinese patient with G114V genetic CJD. Neuropathology 32: 51-59, 2012.

21. Shi XH, Han J, Zhang J, et al: Clinical, histopathological and genetic studies in a family with fatal familial insomnia. Infect Genet Evol 10: 292-297, 2010.

22. Chen JM, Gao C, Shi Q, et al: Different expression patterns of CK2 subunits in the brains of experimental animals and patients with transmissible spongiform encephalopathies. Arch Virol 153: 1013-1020, 2008.

23. Forloni G, Angeretti N, Chiesa R, et al: Neurotoxicity of a prion protein fragment. Nature 362: 543-546, 1993.

24. Florio T, Thellung S, Amico C, et al: Prion protein fragment 106-126 induces apoptotic cell death and impairment of L-type voltage-sensitive calcium channel activity in the GH3 cell line. J Neurosci Res 54: 341-352, 1998.

25. Ettaiche M, Pichot R, Vincent JP and Chabry J: In vivo cytotoxicity of the prion protein fragment 106-126. J Biol Chem 275: 36487-36490, 2000.

26. Drewes G, Trinczek B, IllenbergerS, et al: Microtubule-associated protein/microtubule affinity-regulating kinase (p110mark). A novel protein kinase that regulates tau-microtubule interactions and dynamic instability by phosphorylation at the Alzheimerspecific site serine 262. J Biol Chem 270: 7679-7688, 1995.

27. Manning G, Whyte DB, Martinez R, Hunter T and Sudarsanam S: The protein kinase complement of the human genome. Science 298: 1912-1934, 2002.

28. Moroni RF, De Biasi S, Colapietro P, Larizza L and Beghini A: Distinct expression pattern of microtubule-associated protein/ microtubule affinity-regulating kinase 4 in differentiated neurons. Neuroscience 143: 83-94, 2006.

29. Tagliavini F, Prelli F, Verga L, et al: Synthetic peptides homologous to prion protein residues 106-147 form amyloid-like fibrils in vitro. Proc Natl Acad Sci USA 90: 9678-9682, 1993.

30. Liu YH, Han YL, Song J, et al: Heat shock protein 104 inhibited the fibrillization of prion peptide 106-126 and disassembled prion peptide 106-126 fibrils in vitro. Int J Biochem Cell Biol 43: 768-774, 2011.

31. Magnani I,Novielli C, Bellini M, Roversi G, Bello L and Larizza L: Multiple localization of endogenous MARK4L protein in human glioma. Cell Oncol 31: 357-370, 2009.

32. Terry RD: The pathogenesis of Alzheimer disease: an alternative to the amyloid hypothesis. J Neuropathol Exp Neurol 55: 1023-1025, 1996.

33. Mandelkow EM, Biernat J, Drewes G, Gustke N, Trinczek B and Mandelkow E: Tau domains, phosphorylation, and interactions with microtubules. Neurobiol Aging 16: 355-363, 1995.

34. Zungu M, Schisler JC, Essop MF, McCudden C, Patterson C and Willis MS: Regulation of AMPK by the ubiquitin proteasome system. Am J Pathol 178: 4-11, 2011.

35. Al-Hakim AK, Zagorska A, Chapman L, Deak M, Peggie M and Alessi DR: Control of AMPK-related kinases by USP9X and atypical Lys(29)/Lys(33)-linked polyubiquitin chains. Biochem J 411: 249-260, 2008.

36. Satoh J, Obayashi S, Misawa T, Sumiyoshi K, Oosumi K and Tabunoki H: Protein microarray analysis identifies human cellular prion protein interactors. Neuropathol Appl Neurobiol 35: 16-35, 2009.

37. Stamer K, Vogel R, Thies E, Mandelkow E and Mandelkow EM: Tau blocks traffic of organelles, neurofilaments, and APP vesicles in neurons and enhances oxidative stress. J Cell Biol 156: 1051-1063, 2002.

38. Ebneth A, Godemann R, Stamer K, Illenberger S, Trinczek B and Mandelkow E: Overexpression of tau protein inhibits kinesindependent trafficking of vesicles, mitochondria, and endoplasmic reticulum: implications for Alzheimer's disease. J Cell Biol 143: 777-794, 1998. 